\title{
Rezension zu: Sautter, Hermann: Verantwortlich wirtschaften: Die Ethik gesamtwirtschaftlicher Regelwerke und des unternehmerischen Handelns
}

Reinhard Haupt*

ehem. Universität Jena, Jena

Mit diesem Grundlagenwerk widmet sich der Autor der Aufgabe, Gleichgewichtspfade zwischen ökonomischer Rationalität und moralischer Normativität für verschiedenste wirtschaftliche Themenfelder zu bahnen: Sollensansprüche der Ethik haben sich mit Effizienzerfordernissen der Ökonomik abzustimmen und umgekehrt; erstere stellen wirtschaftliche Sachgesetzlichkeiten, letztere normative Imperative in Frage. Diese holistische Sicht wehrt sich gegen eine „Immunisierung“ sowohl von moralischen Postulaten gegenüber dem ökonomischen Erfolg als auch umgekehrt (S. 24 f., 162). Wirtschaftsparadigmen, die für eine Dominanz des einen über den anderen Bereich stehen, bleiben für Sautter konsequenterweise außerhalb seiner den ökonomischen Wert und ethische Werte integrierenden Perspektive, nämlich einerseits die Fundamentalkritik von Ulrich an der Ökonomik und andrerseits die Moralökonomie von Homann - ersterer Ansatz ein Beispiel für eine Untergewichtung, letzterer für eine Übergewichtung wirtschaftlicher Ansprüche (S. 177 ff.).

Die Strukturierung des Bandes in die zweiHauptteile B und $C$ folgt der Schwerpunktsetzung in eine gesamtwirtschaftliche Grundlegung der Institutionenethik (B) und einzelwirtschaftliche Handlungsmöglichkeiten der Individualethik (C). In Anlehnung an Homann geht es also einerseits um die ordnungsbedingten „Spielregeln“ und andrerseits um die unternehmensbezogenen „Spielzüge“ innerhalb der Regelsysteme.

Zum einen bremst diese Unterscheidung eine vorschnelle Moralisierung über alltägliche Ethikversäumnisse aus: Von Rahmenbedingungen der Gesamtwirtschaft, wie rechtlichen Regelungen oder wirtschaftspolitischen Steuerungen, können wesentliche Anreize für oder gegen wirtschaftsethisches Verhalten ausgehen. Moralisches Versagen ist oft nicht nur einem persönlichen, überzeugungsethischen Fehlverhalten anzulasten, sondern kann auch einem Mangel an Ordnungs-, System- oder Anreizethik geschuldet sein. Die Ausführungen zur Finanzmarktkrise der Jahre 2007/08 (Kap. 9.) betonen z. B., wie sehr Systemfehler der Finanzarchitektur, z. B. laxe MindesteigenkapitalVorschriften für Banken oder die Risiken verschleiernden Techniken zur Kreditverbriefung, mit zu ethischen und wirtschaftlichen Verwerfungen geführt haben.

Zum anderen unterstützt die gesonderte Auseinandersetzung mit der ethischen Dimension einer gesamtwirtschaftlichen Regelsetzung (Teil B) die Erkenntnis, dass auch Normen und Überzeugungen von handelnden Personen diese Ordnungssysteme der Gesamtwirtschaft prägen: Legislative, exekutive und judikative 
Institutionen legen nicht nur Rahmenbedingungen fest, sondern bringen diese gegebenenfalls auch durch mehr oder weniger verantwortungsgebundenes Verhalten, durch Machtungleichgewichte und Informationsasymmetrien, durch Lobbyismus oder politische Mehrheiten hervor (S. 194). So nuanciert Sautter die geläufige Metapher der Homann-Schule („Der systematische Ort der Moral sind die Rahmenbedingungen“) entscheidend: „Der systematische Ort der Moral [...] ist das moralische Handeln [...] und nicht die institutionelle Ordnung, die zwar das menschliche Handeln beeinflusst, selbst aber das Ergebnis menschlichen Handelns ist" (S. 36).

Die Schrift beeindruckt durch manche geschichtliche Spuren, die der Verfasser für die Beantwortung heutiger Fragen zur wirtschaftlichen Verantwortung freilegt. So wird mit der „Tugendethik“ von Aristoteles ein Blick auf das Leitbild einer Kompatibilität von unternehmerischer Kompetenz und moralischer Verantwortung, kurz: von Erfolg und Ethik, geworfen (S. 137) - ein Paradigma, das heute mit dem Vorbild des hansischen „Ehrbaren Kaufmanns" wieder öffentlich thematisiert wird. Auch der Schumpeter'sche „dynamische Unternehmer" (z. B. konkretisiert in der Person des Apple-Gründers Steve Jobs, S. 83) oder die „visionary company“ (etwa am Beispiel des Familienunternehmens Sennheiser, S. 564) spiegeln etwas von dem aristotelischen Ethos eines sachbezogenen Strebens nach Produktqualität und Innovationsexzellenz wider - im Gegensatz zur „unnatürlichen Erwerbskunst“ einer vordergründig finanzgetriebenen Unternehmung (S. 80 f.).

Das Werk überzeugt immer wieder durch die Ausgewogenheit seiner Argumentation in manchen strittigen Fragen des gegenwärtigen Wirtschaftslebens. Am Beispiel des flächendeckenden Mindestlohns werden z. B. Begründungen (Anomalien des Arbeitsangebots, monopsonistische Arbeitsnachfrage) und Vorbehalte (Marktinkonformität) gegeneinander abgewogen (S. 341 ff.). Ebenso tritt Sautter etwa der leichtfertigen Brandmarkung der „Schwarzen Kassen“ in der jüngeren Siemens-Vergangenheit durch eine Beschäftigung mit den Motiven und Zwängen eines im globalen Wettbewerb stehenden Players entgegen (S. 30 ff.). Ähnliches gilt für ein so aufgeladenes Thema wie die Legalisierung des Organhandels: Außer mit den ethischen Vorbehalten wird der Leser auch mit der Überzeugungskraft einer Marktregelung vertraut gemacht, die schließlich in einer Art „Club"-Lösung (Vorrang von Organspendern bei der Organzuteilung) einen ethisch und ökonomisch erträglichen Kompromiss findet (S. $281 \mathrm{ff}$.$) .$

Dezidiert anders als manche Ökonomen ordnet Sautter die „Moralökonomie“ Homanns als einen „alternativen" Wirtschaftsethik-Ansatz ein, der den Willen zur
Integration von Effizienzerfordernissen mit Normativitätsansprüchen vermissen lässt. In der Tat stehen in der Ethiksicht von Homann und seinen Schülern die utilitaristischen Eigeninteressen und das Vorteilsdenken im Mittelpunkt, wenn sie auch am Rande zugestehen, dass die Überzeugungsethik nicht ersetzt, sondern durch einen anreizgestützten Impuls gestärkt werden soll (Homann et al. 2005, S. 89 f.). (Diese Sicht wird auch von Hax, einem Protagonisten der Homann-Community, geteilt (Hax 1993, S. 776) - dessen Beitrag übrigens erstaunlicherweise in diesem sonst so quellenreichen Band keine Berücksichtigung findet.)

Man könnte sich vielleicht wünschen, dass der Autor die Nischen einer win-win-Situation zwischen Moral und Ökonomie, den Fall der „positiven Kompatiblität“ (S. 569) bzw. den „business case“ (S. 605 ff.), positiver gewürdigt hätte. Wer wollte es dem in Wirtschaft und Unternehmen verantwortlich Handelnden verdenken, wenn er sich vorrangig Managementfeldern und Anwendungsfällen widmet, die ihm ein positives ökonomisches Signal seines Ethikverhaltens unmittelbar rückmelden? Die Erfahrung des „doing well by doing good“ kann ein Einstieg in ein ethisches Bewusstsein bedeuten und die Akzeptanz von Normativitätsansprüchen fördern.

Man muss auch nicht die Skepsis von Sautter gegenüber einer ökologischen Verträglichkeit eines innovationsgetriebenen Wachstums teilen (S. 409 ff.). Der Trend zu einer Dematerialisierung der Wirtschaft ist jedenfalls unübersehbar, wenn man an die Tertiarisierung des Sozialprodukts, d.h. das Wachstum der dienstleistenden zu Lasten der Sachgüter-Branchen, denkt. Vor allem hat die Zukunft der Digitalisierung, die Produktivitätsfortschritte praktisch ohne zusätzlichen Ressourcenverbrauch verheißt, gerade erst begonnen und dürfte Zweifel an einer Postwachstumsökonomie unterstützen.

Schließlich erscheinen die Vorbehalte des Autors gegenüber der Lösung des Energie- und Klimaproblems durch Emissionslizenzen umstritten (S. 393 ff.). Selbst wenn man das europäische Emissions Trading Scheme (ETS) wegen der Branchenausnahmen und des Ausschlusses des Verkehrswesens für unbefriedigend hält, bietet es europaweit im Vergleich zum Erneuerbare Energien-Gesetz (EEG) eine überzeugendere Emissionsbilanz. Im weltweiten Maßstab könnte ein globales ETS Vorbildcharakter gewinnen und sich den Selbstbindungsregeln nach dem Pariser Klimaschutzabkommen von 2015 als überlegen erweisen, da diese durch Rückzugsoptionen ohne Sanktionen Zweifel an ihrer Stabilität wecken.

Solche Einschränkungen können aber nicht den souveränen Eindruck verwischen, den das Gesamtwerk hinterlässt. Nach Breite und Tiefe der 
Argumentation, nach Quantität und Qualität des Stoffumfangs wird es Vorbildcharakter für zukünftige Monographien zur Wirtschafts- und Unternehmensethik haben. Es gelingt Sautter auch mit seiner anschaulichen Stoffpräsentation, den Leser durch seine Argumentation zu führen: Die vielfachen Einführungen und Résumés zum jeweiligen Kapitel-/ Abschnitt-Anfang/Ende unterstützen die Orientierung und das Verständnis bei der Lektüre.

Mit diesem Werk setzt der Verfasser Maßstäbe einer umfassenden, überzeugenden, ausgewogenen und aktuellen Auseinandersetzung mit Begründungen und Ansprüchen, mit Ergebnissen und Defiziten von Verantwortung bei Entscheidungsträgern ökonomischer Institutionen. Man wird im gegenwärtigen wirtschaftswissenschaftlichen Fachschrifttum schwerlich ein vergleichbar anspruchsvolles und gründliches Kompendium zur Wirtschafts- und Unternehmensethik finden. Allein das quantitative Volumen dieser Forschungsleistung setzt Benchmarks für eine wissenschaftliche Studie: Auf über 800 Seiten werden annähernd 1.000 Literaturquellen, viele zeitgenössische Brennpunktbeobachtungen des Wirtschaftslebens sowie bis in die allerjüngste Gegenwart reichende Daten und Fakten des ökonomischen Alltags herangezogen und ausgewertet. Man möchte ohne Übertreibung von einem Meilenstein wirtschaftswissenschaftlicher Forschung sprechen, das sich dem Spannungsfeld zwischen Markt und Moral oder zwischen Gewinn und Gewissen widmet. 